УДК 546.719:621.793.1

\author{
M. S. Iziumskyi, A. V. Shtemenko \\ Ukrainian State University of Chemical Technology, Department of inorganic chemistry, \\ 8 Gagarin avenue, Dnipro, 49005, Ukraine, \\ email: maksimizumsky@gmail.com
}

\title{
RADICAL MECHANISM OF FORMATION OF CU-Re AND Pb-Re COMPOSITES IN GASEOUS PHASE BY THERMAL DECOMPOSITION OF TRANS-TETRACHLORODI- $\mu-$ PROPIONATO DIRHENIUM(III)
}

\begin{abstract}
Reactions of individual trans-tetrachloro-di- $\mu$-propionato dirhenium(III) thermal decomposition in dynamic inert atmosphere were investigated. Using a radical reaction of transportation in gaseous phase, $\mathrm{Cu}-\mathrm{Re}$ and $\mathrm{Pb}-\mathrm{Re}$ composites were obtained on a ceramic surface. Free radicals $\mathrm{C}_{2} \mathrm{H}_{5} \cdot$ were detected by reaction with metal $\mathrm{Cu}$ and $\mathrm{Pb}$ mirrors. $\mathrm{Cu}-\mathrm{Re}$ and $\mathrm{Pb}-\mathrm{Re}$ composites were studied by $\mathrm{XRD}$, micro-X-ray spectral and SEM analysis. $\mathrm{Cu}-\mathrm{Re}$ composites contain nano- dendrites of $\mathrm{Cu}$ which «grow» from a Re base and most likely have a nanotube structure. Dimensions of the nanotubes can vary from $100 \mathrm{~nm}$ to $1 \mu \mathrm{m}$.
\end{abstract}

Keywords: rhenium, free radical, carboxylate, composite, mechanism, decomposition.

Binuclear cluster carboxylates of rhenium(III), obtained for the first time by A.S. Kotel'nikova (USSR) and Cotton (USA), are the classical complexes in chemistry of clusters [1-4]. Currently information related to the radical mechanism of thermal destruction of dirhenium(III) complexes and forming $\mathrm{Cu}-\mathrm{Re}$ and $\mathrm{Pb}$ - $\mathrm{Re}$ composites is not available.

The current understanding of the chemistry of rhenium carboxylates is reviewed in the book «Multiple bonds between metal atoms» [3].

Investigation of thermal decomposition is very important for the determination of a detailed radical mechanism of reactions and detecting free $\mathrm{C}_{2} \mathrm{H}_{5} \cdot$ radical by interaction with metals like $\mathrm{Cu}$ and $\mathrm{Pb}$. It provides an opportunity to obtain very pure Rhenium metal for a specialist industry, Re coatings and new composite materials using a chemical vapor deposition $[5,6]$.

In this article we obtained $\mathrm{Cu}-\mathrm{Re}$ and $\mathrm{Pb}-\mathrm{Re}$ composites by reaction of free $\mathrm{C}_{2} \mathrm{H}_{5} \cdot$ radical with a $\mathrm{Pb}$ mirror and compact Copper. Free $\mathrm{C}_{2} \mathrm{H}_{5}$ - radicals were formed as a result of thermal decomposition of trans-tetrachloro-di- $\mu$-propionato dirhenium(III).

\section{RESULTS AND DISCUSSION}

Ceramic-based Copper-Rhenium and Lead-Rhenium composites were obtained using thermal decomposition of trans-tetrachloro-di- $\mu$-propionato dirhenium(III) in a quartz tube (Fig. 1).

During the procedure trans-tetrachloro-di- $\mu$-propionato dirhenium(III) evaporates in a stream of inert gas at $300^{\circ} \mathrm{C}$ [7] on heater 1 followed by thermal decomposition on $\mathrm{Cu}$ or $\mathrm{Pb}$ surface at $800^{\circ} \mathrm{C}$ on heater 2 . We carried out three experiments using different process conditions. Thermal decomposition of trans-tetrachloro-di- $\mu$-propionato dirhenium(III) in air was studied in our previous work [6]. 


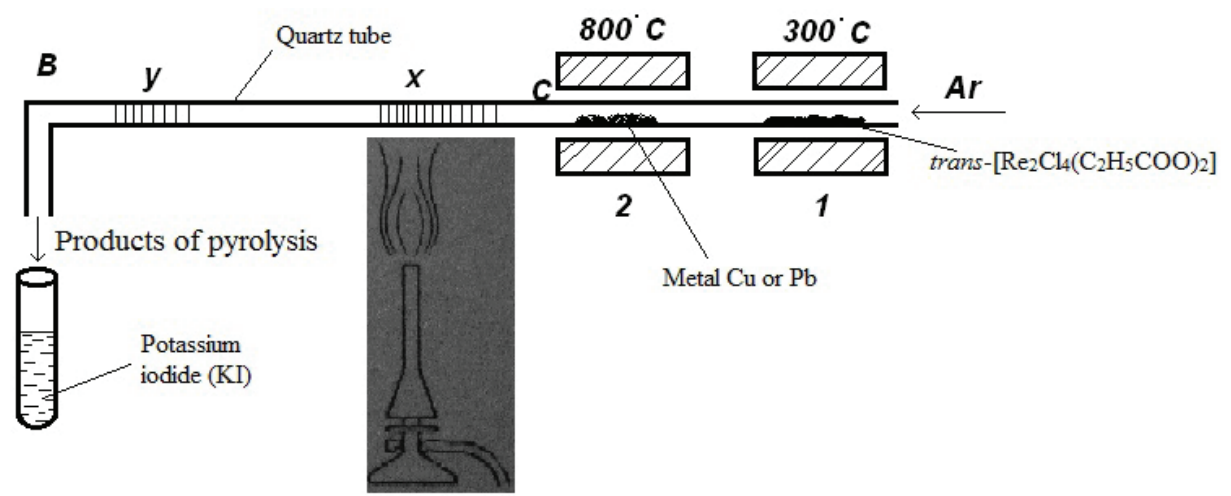

Fig. 1. Experimental set-up used for conformation of free-radical thermal decomposition of trans-tetrachloro-di- $\mu$-propionato dirhenium(III) and formation of metal composites.

Experiment $1.1 \mathrm{~g}$ pure trans-tetrachloro-di- $\mu$-propionato dirhenium(III) was placed in zone 1 of a quartz tube (Fig. 1). Zone 2 was free. Re-mirror appeared after 5 hrs heating in zone 2 (Fig. 2). Metal rhenium was detected by XRD analysis.

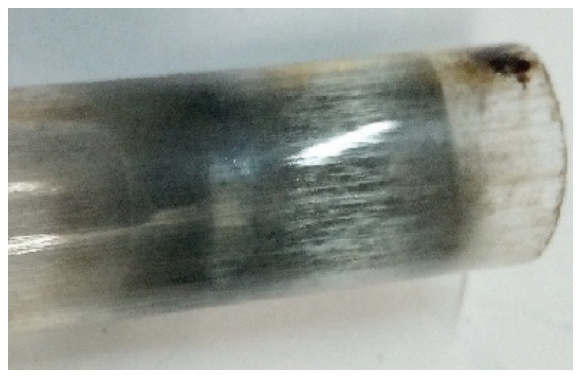

a)

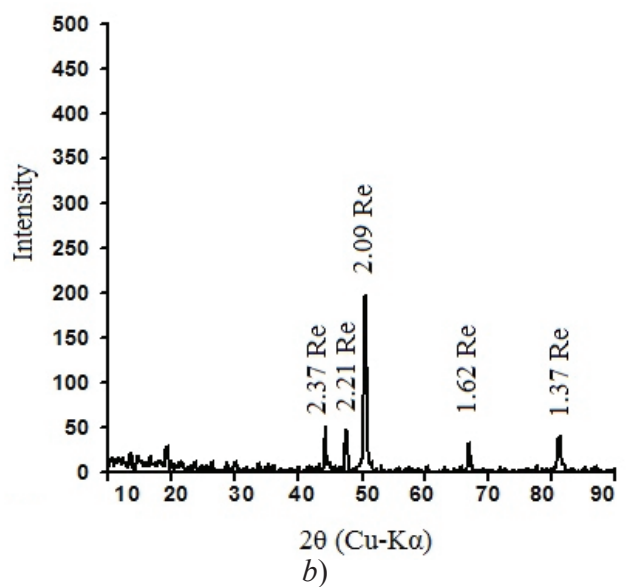

Fig. 2. Rhenium mirror $(a)$ and X-ray diffraction $(b)$ of a deposit formed in a quartz tube. DRON-3, $\mathrm{Cu}-\mathrm{K} \alpha$-radiation.

Quantity of produced Re was detected by gravimetric method from a mass increase of the quartz tube. On the next stage, $\mathrm{Re}$ was dissolved in $\mathrm{H}_{2} \mathrm{O}_{2}$. Perrhenic acid $\left(\mathrm{HReO}_{4}\right)$ was detected (tan coloured substance) by mixing with potassium thiocyanate (KSCN) and tin(II) chloride $\left(\mathrm{SnCl}_{2}\right)$ [8]. $\mathrm{HReO}_{4}$ was titrated using sodium hydroxide $(\mathrm{NaOH})$ and a phenolphthalein indicator [8]. Quantity of the obtained rhenium was $99 \%$ by mass of the original trans-complex. Carbon (IV) dioxide $\left(\mathrm{CO}_{2}\right)$ was detected by reaction with calcium hydroxide $\left(\mathrm{Ca}(\mathrm{OH})_{2}\right)$. Hypothetical halogenalkyls $\left(\mathrm{C}_{2} \mathrm{H}_{5} \mathrm{Cl}\right.$ and $\left.\mathrm{C}_{2} \mathrm{H}_{4} \mathrm{Cl}_{2}\right)$ were dissolved in two different solvents: (tetrachloromethane (solution 1) and dimethyl ketone (solution 2)). 1-2 ml of solution 1 was added to a tube followed by addition of 10-20 $\mathrm{mg}$ of sodium thiosulfate $\left(\mathrm{Na}_{2} \mathrm{~S}_{2} \mathrm{O}_{3}\right)$. The tube was placed in a glycerine bath and kept 
at $180^{\circ} \mathrm{C}$. the tube top was paper-covered with an infused reagent (congo indicator with hydrogen peroxide). The colour of the paper has changed to blue [9]. 1-2 ml of solution 2 was added to a microtube followed by addition of $2 \mathrm{ml}$ pyridine and $5 \mathrm{~N}$ sodium hydroxide. The colour of pyridine has changed to light pink.

Thermal decomposition of trans-tetrachloro-di- $\mu$-propionato dirhenium(III) in a stream of inert gas runs according to Schematic 1.

$$
\begin{gathered}
\mathrm{Re}_{2} \mathrm{Cl}_{4}\left(\mathrm{C}_{2} \mathrm{H}_{5} \mathrm{COO}\right)_{2} \rightarrow 2 \mathrm{Re}+4 \mathrm{HCl}+\mathrm{C}_{2} \mathrm{H}_{2}+\mathrm{C}_{2} \mathrm{H}_{4}+2 \mathrm{CO}_{2} \\
\mathrm{HC} \equiv \mathrm{CH}+\mathrm{HCl} \rightarrow \mathrm{H}_{2} \mathrm{C}=\mathrm{CHCl}+\mathrm{HCl} \rightarrow \mathrm{H}_{3} \mathrm{C}-\mathrm{CH}_{2} \mathrm{Cl} \\
\mathrm{C}_{2} \mathrm{H}_{4}+\mathrm{HCl} \rightarrow \mathrm{C}_{2} \mathrm{H}_{5} \mathrm{Cl} \\
\mathrm{Re}_{2} \mathrm{Cl}_{4}\left(\mathrm{C}_{2} \mathrm{H}_{5} \mathrm{COO}\right)_{2} \rightarrow 2 \mathrm{Re}+4 \mathrm{HCl}+\mathrm{C}_{2} \mathrm{H}_{5} \mathrm{Cl}+\mathrm{C}_{2} \mathrm{H}_{4} \mathrm{Cl}_{2}+2 \mathrm{CO}_{2}
\end{gathered}
$$

Scheme 1. Thermal decomposition at $800^{\circ} \mathrm{C}$ of trans-tetrachloro-di- $\mu$-propionato dirhenium(III) in a stream of inert gas.

Experiment 2. $1 \mathrm{~g}$ pure trans-tetrachloro-di- $\mu$-propionato dirhenium(III) was placed in zone 1 of the quartz tube (Fig. 1). At the same time, $0.1 \mathrm{~g}$ metal $\mathrm{Cu}$, ceramic $\left(\mathrm{Mg}_{2} \mathrm{Al}_{4} \mathrm{Si}_{6} \mathrm{O}_{18}\right)$ and a quartz plate were placed in zone 2 of the quartz tube. A CopperRhenium composite was obtained after 5 h heating in the end of zone 2 (Fig. 3) and detected by XRD analysis.

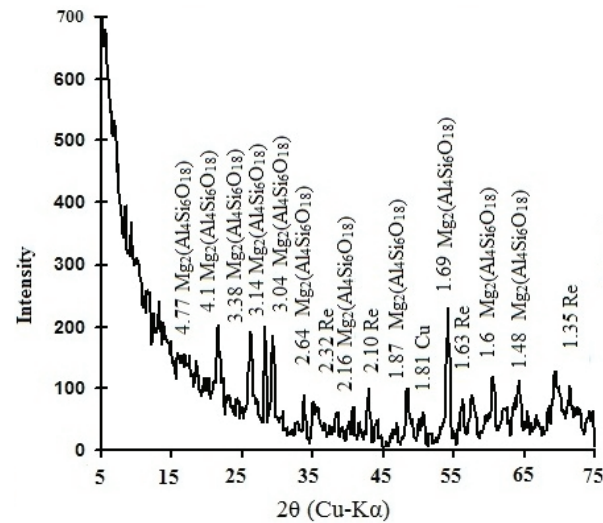

a)

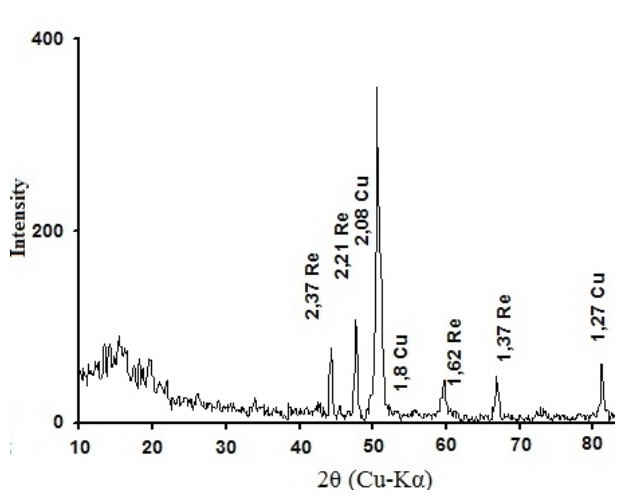

b)

Fig. 3. X-ray diffraction of Copper-rhenium composite formed on cordierite $\left(\mathrm{Mg}_{2} \mathrm{Al}_{4} \mathrm{Si}_{6} \mathrm{O}_{18}\right)(a)$ and quartz tube $(b)$. DRON-3, $\mathrm{Cu}-\mathrm{K} \alpha$-radiation.

The halogenalkyls were analyzed in the same way as described in Experiment 1. Because of instability of $\mathrm{Cu}\left(\mathrm{C}_{2} \mathrm{H}_{5}\right)_{2} \uparrow$ or $\mathrm{Cu}\left(\mathrm{C}_{2} \mathrm{H}_{5}\right)_{n} \uparrow$, their presence is theoretically based on the publications data [10-19]. As a matter of fact, $\mathrm{Cu}\left(\mathrm{C}_{2} \mathrm{H}_{5}\right)_{n} \uparrow$ cannot be obtained in a solid state. The reaction of thermal decomposition of trans-tetrachloro-di- $\mu$-propionato dirhenium(III) with metal $\mathrm{Cu}$ in a stream of inert gas runs according to Schematic 2.

$$
\begin{gathered}
\text { trans }-\left[\mathrm{Re}_{2} \mathrm{Cl}_{4}\left(\mathrm{C}_{2} \mathrm{H}_{5} \mathrm{COO}\right)_{2}\right] \uparrow \rightarrow 2 \mathrm{Re} \downarrow+2 \mathrm{C}_{2} \mathrm{H}_{5} \bullet \uparrow+2 \mathrm{CO}_{2} \uparrow+4 \mathrm{Cl} \uparrow \uparrow \\
\mathrm{Cu} \downarrow+2 \mathrm{C}_{2} \mathrm{H}_{5} \bullet \uparrow \rightarrow \mathrm{Cu}\left(\mathrm{C}_{2} \mathrm{H}_{5}\right)_{2} \uparrow
\end{gathered}
$$




$$
\begin{gathered}
\text { trans- }\left[\mathrm{Re}_{2} \mathrm{Cl}_{4}\left(\mathrm{C}_{2} \mathrm{H}_{5} \mathrm{COO}\right)_{2}\right] \uparrow+\mathrm{Cu}\left(\mathrm{C}_{2} \mathrm{H}_{5}\right)_{2} \uparrow \rightarrow \mathrm{Cu}-2 \mathrm{Re} \downarrow+4 \mathrm{C}_{2} \mathrm{H}_{5} \bullet \uparrow+4 \mathrm{Cl} \bullet \uparrow+2 \mathrm{CO}_{2} \uparrow \\
4 \mathrm{C}_{2} \mathrm{H}_{5} \bullet \uparrow+4 \mathrm{Cl} \bullet \uparrow \rightarrow 4 \mathrm{C}_{2} \mathrm{H}_{5} \mathrm{Cl} \uparrow
\end{gathered}
$$

Scheme 2. Thermal decomposition of trans-tetrachloro-di- $\mu$-propionato dirhenium(III) with metal $\mathrm{Cu}$ in a stream of inert gas.

Different phases of $\mathrm{Cu}$ and $\mathrm{Re}$ can be clearly seen from Fig. 3. SEM images of $\mathrm{Cu}-$ Re composite are shown in Fig. 4. Images $g$ and $h$ in Fig. 4 show dendrites of $\mathrm{Cu}$ that «grow» from a Re base and most likely have a nanotube structure. Dimensions of the nanotubes can vary from $100 \mathrm{~nm}$ to $1 \mu \mathrm{m}$ Micrographs in Fig. 4 demonstrate a complex

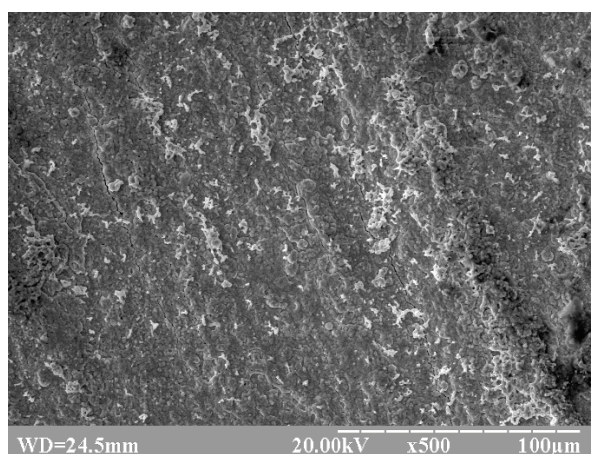

a)

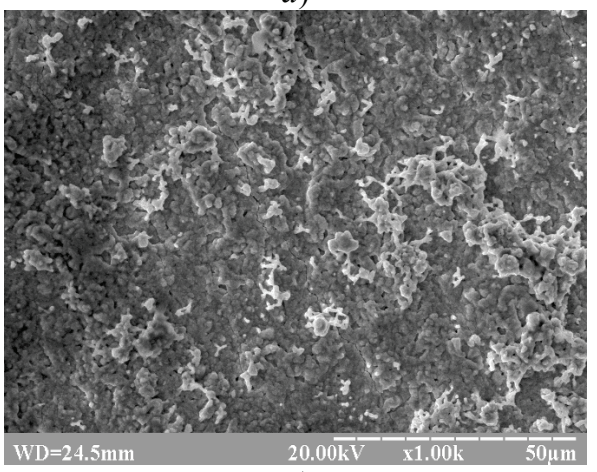

c)

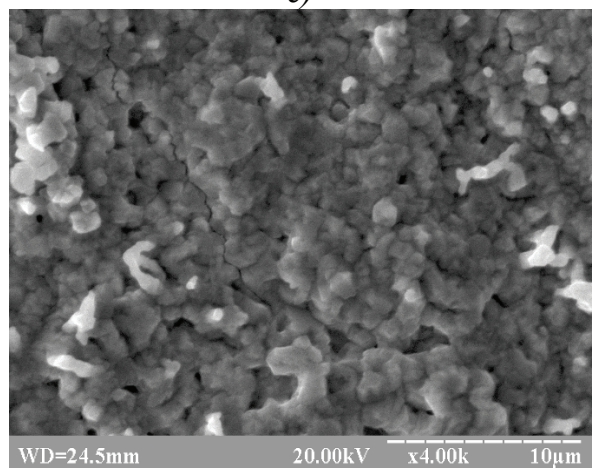

e)

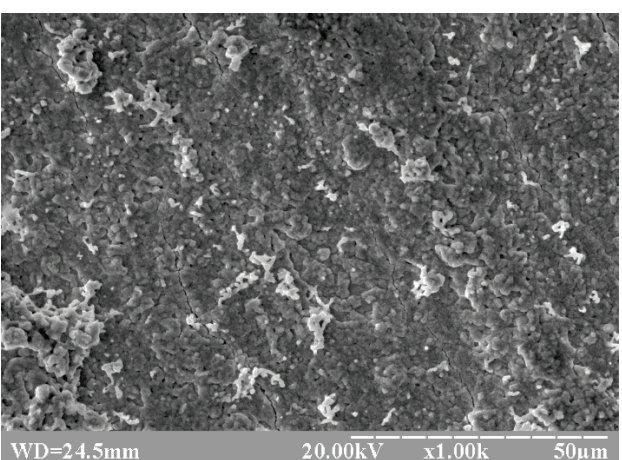

b)

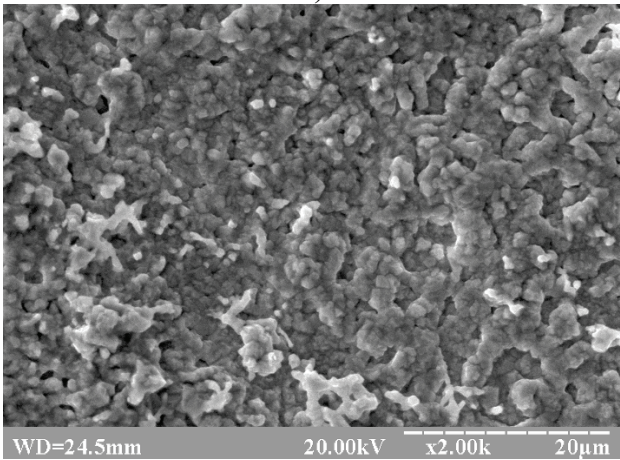

d)

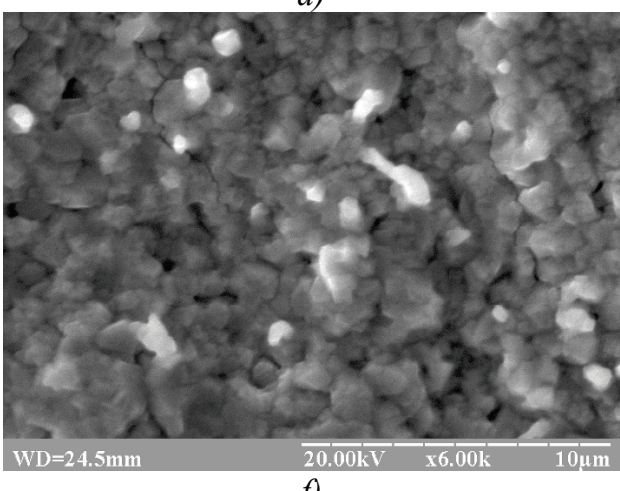

f) 


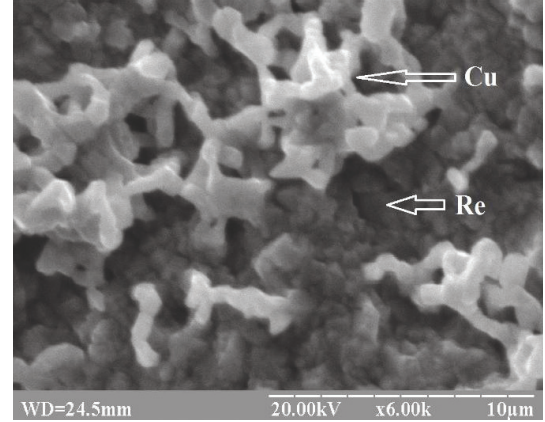

g)

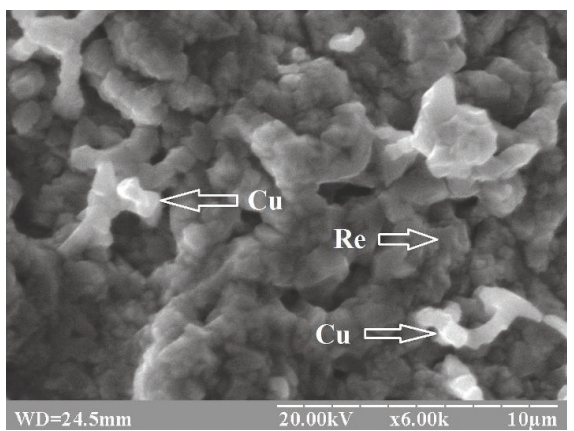

h)

Fig. 4. SEM images of $\mathrm{Cu}$-Re deposits formed on cordierite $\left(\mathrm{Mg}_{2} \mathrm{Al}_{4} \mathrm{Si}_{6} \mathrm{O}_{18}\right)$. $\mathrm{Re}$ and $\mathrm{Cu}$ were detected by micro-X-ray spectral analysis.

structure of crystals with dimensions from $0.5 \mu \mathrm{m}$ to $20 \mu \mathrm{m}$. Their composition was confirmed by micro-X-ray spectral analysis. Co-deposition of $\mathrm{Re}$ and $\mathrm{Cu}$ was carried out using a non-metallic substrate in order to eliminate red-ox reactions with $\mathrm{Fe}$ or other active metals.

Experiment 3. $1 \mathrm{~g}$ pure trans-tetrachloro-di- $\mu$-propionato dirhenium(III) was placed in zone 1 of the quartz tube (Fig. 1). At the same time, $0.1 \mathrm{~g}$ metal $\mathrm{Pb}$, was placed in zone 2 of the quartz tube. Lead-Rhenium composite was obtained after $5 \mathrm{hrs}$ heating in the end of zone 2 (Fig. 1 and Fig. 5). Lead-rhenium composite was detected by XRD analysis.

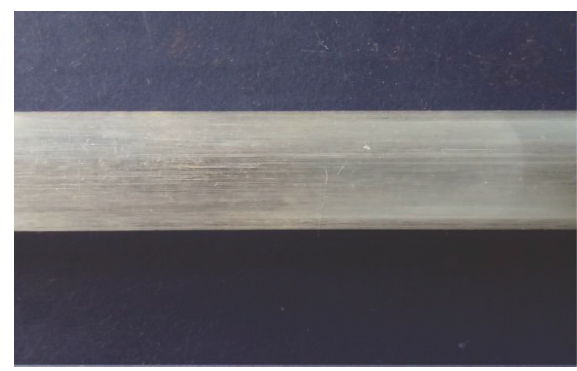

a)

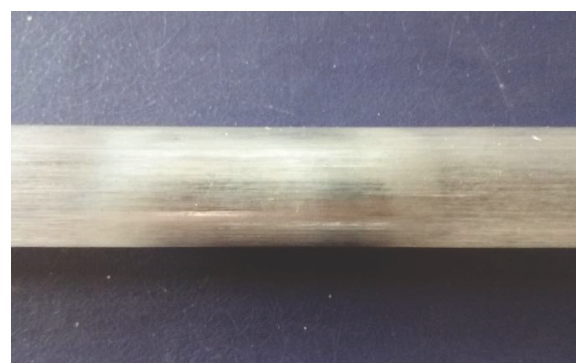

b)

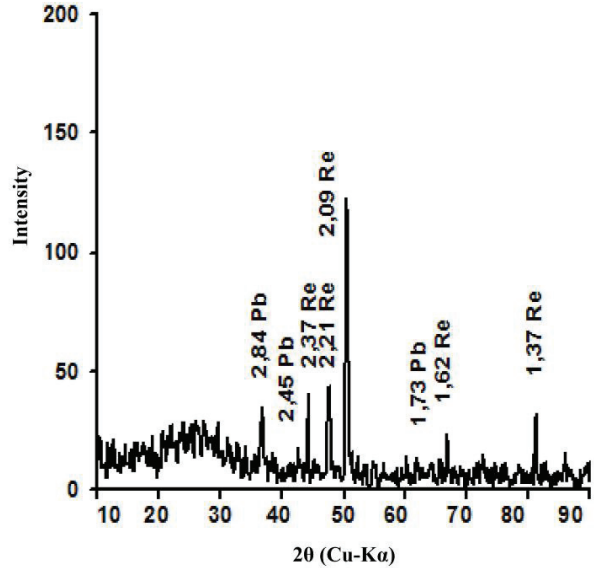

c)

Fig. 5. Lead-rhenium composite and its XRD analysis.

a) Photo of a quartz tube before deposition.; b) Photo of a quartz tube after deposition. 
After removal of $\mathrm{Pb}$ from zone 2 and heating zone $\mathbf{x}$ and zone $\mathbf{y}$ in a stream of gaseous mixture of trans-tetrachloro-di- $\mu$-propionato dirhenium(III) and argon pure metal Re was obtained in zone $\mathbf{x}$ (Fig. 6) and Pb-Re composite - in zone $\mathbf{y}$.

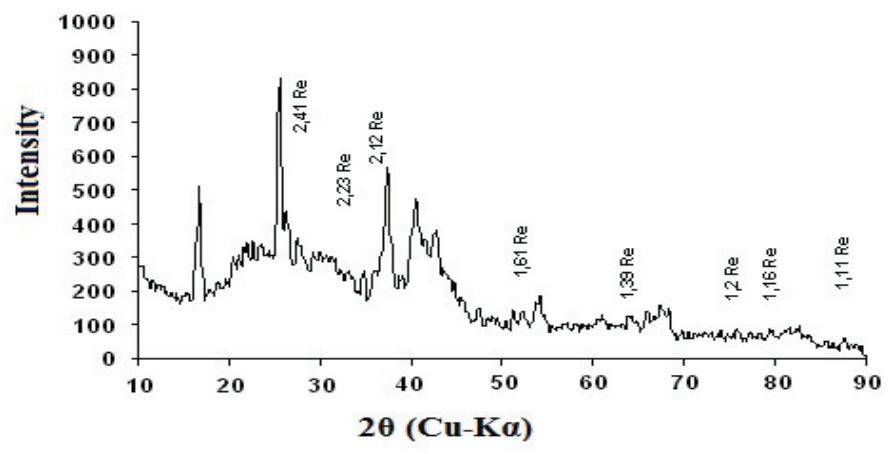

Fig. 6. X-ray diffraction of Rhenium formed in zone $\mathbf{x}$.

Formation of yellow precipitate took place after gaseous mixture passed through a solution of potassium iodide (KI), with further addition of sodium thiosulfate. XRD analysis of yellow precipitate is shown in Fig. 7.

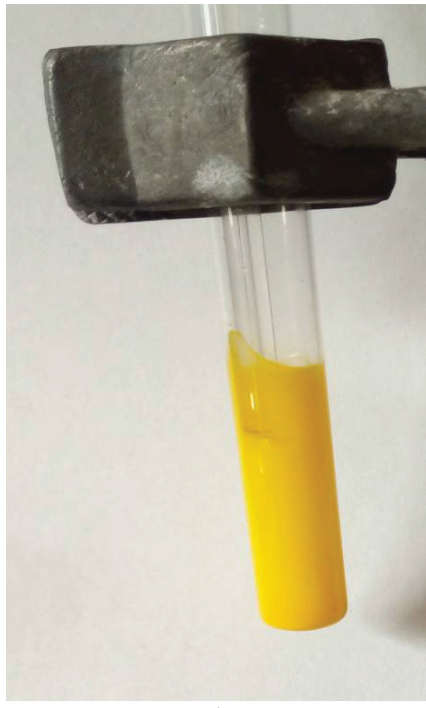

a)

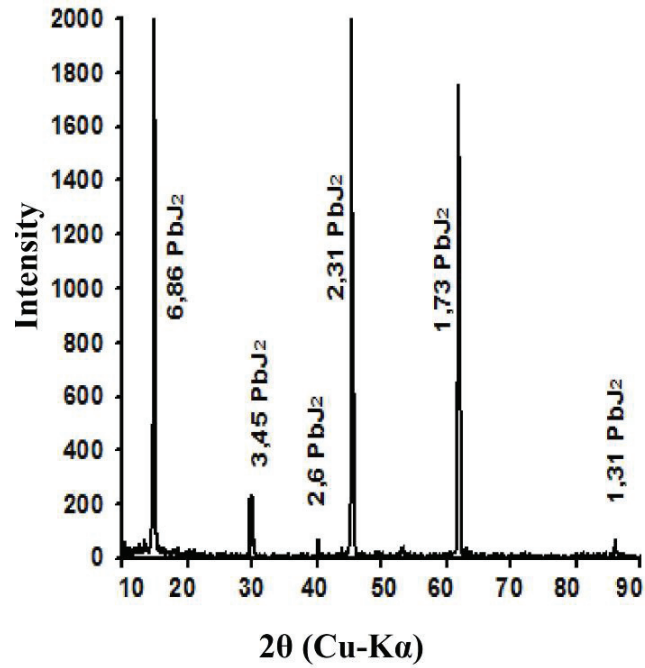

b)

Fig. 7. X-ray diffraction of yellow precipitate.

The halogenalkyls were analyzed in the same way as described in Experiment 1 . The reaction of thermal decomposition of trans-tetrachloro-di- $\mu$-propionato dirhenium(III) with metal $\mathrm{Pb}$ in a stream of inert gas runs according to Schematic 3.

Knowledge about formation of $\mathrm{Pb}\left(\mathrm{C}_{2} \mathrm{H}_{5}\right)_{4} \uparrow$ or $\mathrm{Pb}\left(\mathrm{C}_{2} \mathrm{H}_{5}\right)_{\mathrm{n}} \uparrow$ is based on previous publications [10-19] and reactions with $\mathrm{KI}$ and metal mirrors. 


$$
\begin{gathered}
\text { trans }-\left[\mathrm{Re}_{2} \mathrm{Cl}_{4}\left(\mathrm{C}_{2} \mathrm{H}_{5} \mathrm{COO}\right)_{2}\right] \uparrow \rightarrow 2 \mathrm{Re} \downarrow+2 \mathrm{C}_{2} \mathrm{H}_{5} \bullet \uparrow+2 \mathrm{CO}_{2} \uparrow+4 \mathrm{Cl} \bullet \uparrow \\
1 / 2 \mathrm{~Pb} \downarrow+2 \mathrm{C}_{2} \mathrm{H}_{5} \bullet \uparrow \rightarrow 1 / 2 \mathrm{~Pb}\left(\mathrm{C}_{2} \mathrm{H}_{5}\right)_{4} \uparrow \\
\text { trans }-\left[\operatorname{Re}_{2} \mathrm{Cl}_{4}\left(\mathrm{C}_{2} \mathrm{H}_{5} \mathrm{COO}\right)_{2}\right] \uparrow+1 / 2 \mathrm{~Pb}\left(\mathrm{C}_{2} \mathrm{H}_{5}\right)_{4} \uparrow \rightarrow 1 / 2 \mathrm{~Pb}-2 \mathrm{Re} \downarrow+4 \mathrm{C}_{2} \mathrm{H}_{5} \bullet \uparrow+4 \mathrm{Cl} \bullet \uparrow+2 \mathrm{CO}_{2} \uparrow \\
4 \mathrm{C}_{2} \mathrm{H}_{5} \bullet \uparrow+4 \mathrm{Cl} \bullet \uparrow \rightarrow 4 \mathrm{C}_{2} \mathrm{H}_{5} \mathrm{Cl} \uparrow
\end{gathered}
$$

Scheme 3. Thermal decomposition of trans-tetrachloro-di- $\mu$-propionato dirhenium(III) with metal $\mathrm{Pb}$ in a stream of inert gas.

\section{EXPERIMENTAL SECTION}

In this research initial $\left.\left[\mathrm{N}\left(n-\mathrm{C}_{4} \mathrm{H}_{9}\right)_{4}\right)\right]_{2}\left[\mathrm{Re}_{2} \mathrm{Cl}_{8}\right]$ and trans-carboxylates were synthesized without using an autoclave. The complexes obtained in the present study have been evaluated by electron absorption and elemental analysis [20-23]. All reagents and solvents are commercially available.

Preparation of compounds. $\left.\left[\mathrm{N}\left(n-\mathrm{C}_{4} \mathrm{H}_{9}\right)_{4}\right)\right]_{2}\left[\mathrm{Re}_{2} \mathrm{Cl}_{8}\right] .2 .0 \mathrm{~g}$ of $\left[\mathrm{N}\left(n-\mathrm{C}_{4} \mathrm{H}_{9}\right)_{4} \mathrm{ReO}_{4}\right.$ was added to $20 \mathrm{ml}$ of benzoylchloride $\left(\mathrm{C}_{6} \mathrm{H}_{5} \mathrm{COCl}\right)$. The solution was heated under reflux in a Nitrogen atmosphere at $210^{\circ} \mathrm{C}$ for 5 hours. Consequently, $3.33 \mathrm{~g}$ of $\left[\mathrm{N}\left(n-\mathrm{C}_{4} \mathrm{H}_{9}\right)_{4} \mathrm{Br}\right.$ was dissolved in $30 \mathrm{ml}$ of saturated with a hydrogen chloride ethanol and added to the solution. This mixture remained under reflux at $210^{\circ} \mathrm{C}$ for $1.5 \mathrm{hrs}$ in a Nitrogen atmosphere. Using filtration, a blue crystalline substance was obtained. It was washed using three $10 \mathrm{ml}$ portions of isopropyl alcohol then ethanol and dried under vacuum. The substance yield is $2.2462 \mathrm{~g}$ or $97 \%$.

Analysis. Calculations for $\mathrm{C}_{32} \mathrm{H}_{72} \mathrm{~N}_{2} \mathrm{Cl}_{8} \mathrm{Re}_{2}$ : C, 33.69; H, 6.36; N, 2.45; Cl, 24.9; Re, 32.65. Found: C, 33.0; H, 6.15; $\mathrm{N}, 2.5 ; \mathrm{Cl}, 24.8$; Re, 32.6. UV-vis (acetonitrile), $\lambda_{\max }, \mathrm{cm}^{-1}: 14700,20940,23645,27000,28100,32600,39215$. [24-26]

The synthesis of trans-tetrachloro-di- $\mu$-propionato dirhenium(III) is reviewed in [6]

Diffractometer DRON-3 with $\mathrm{Cu}-\mathrm{K} \alpha$ radiation was used for X-ray diffraction analysis. Scanning Electron Microscope with Micro-Analyzer SEMMA-102-02 was used to obtain SEM images.

\section{CONCLUSIONS}

Radical mechanism of thermal decomposition of trans-tetrachloro-di- $\mu$-propionato dirhenium(III) was confirmed. Mechanism of free-radical reactions was established by reaction of $\mathrm{C}_{2} \mathrm{H}_{5} \bullet$ with $\mathrm{Cu}$ and $\mathrm{Pb}$ metal mirrors. Microstructure of $\mathrm{Cu}-\mathrm{Re}$ composite was revealed. This research may be used for explanation of the CVD processes while obtaining coatings, composites and new precision materials.

\section{REFERENCES}

1. Kotel 'nicova A.S., Tronev V.G. Research the complexes of rhenium(II) // Zh. Neorg. Khim. - 1958. - Vol. 3. P.1008-1027.

2. Cotton F.A. Metal-Metal Bonding in $\left[\mathrm{Re}_{2} \mathrm{X}_{8}\right]^{2-}$ Ions and Other Metal Atom Clusters. // Inorg. Chem. - 1965. Vol. 4. - P.334-336. https://doi.org/10.1021/ic50025a016

3. Cotton F.A., Murillo C.A., Walton R.A. Multiple bonds between metal atoms. - New York: Springer, 2005. $818 \mathrm{p}$.

4. Steblevsky A.V., Alihanian A.S., Vedenkina L.G. Mass spectral investigation processes of sublimation some rhenium cluster compounds. // Russ. J. Coord. Chem. - 1984. - Vol. 10. - P. 72-76. 
5. Iziumskyi M., Melnyk S., Shtemenko A. Polymetallic Copper-Rhenium Composite Material. // Chem. Met. Alloys. - 2013. - Vol. 6, N 3/4. - P. 121-124.

6. Iziumskyi M., Baskevich A., Melnyk S., Shtemenko A. Thermodynamic properties of trans-tetrachlorodi- $\mu$ carboxylate dirhenium(III) complexes. // New. J. Chem. - 2016. - Vol. 40. - P. 10012-10015.

7. Shtemenko A.V., Bovykin B.A., Shram B.C. Thermal decomposition of rhenium binuclear halocarboxylates. // Zh. Neorg. Khim. - 1985. - Vol. 12, N(30). - P. 3085-3089.

8. Borisova L.V., Ermakov A.N. Analytical chemistry of rhenium. - Moscow: Science, 1974. - 319p.

9. Feigl F. Spot tests in organic analysis (6th ed.). - New York: Elsevier, 1960. -836p.

10. Rozancev E.G., Sholle V.D. Organic chemistry of free radicals. - Moscow: Chemistry, 1979. - 344 p.

11. Cotton F.A., Wilkinson G. Advanced inorganic chemistry. - Willey, 1969. - 592p.

12. Cotton F.A., Wilkinson G. Basic inorganic chemistry. - Willey, 1976. - 678p.

13. Green M. Organometallic compounds. The transition elements. Vol. 2. - London, 1968. -457 p.

14. Kostromina N.A., Kumok V.N., Skorik N.A. Chemistry of coordination compounds. - Moskow: High School, 1990. $-432 \mathrm{p}$

15. Alekseev S.O. Chemistry of complex compounds. - Kiev, 2010. -159 p.

16. Lawrance G.A. Introduction to coordination chemistry. - Willey, 2010. - $290 \mathrm{p}$.

17. Pohodenko V.D., Beloded A.A., Koshechko V.G. Redox reactions of free radicals. - Kiev: Scientific idea, 1977. $277 \mathrm{p}$.

18. Sokolov M.N., Samsonenko D.G. Coordination chemistry. Part II: Metalorganic compounds, catalysis with complexes of transition metals, cluster compounds. - Novosibirsk, 2011. - $194 \mathrm{p}$.

19. Zeiss H. Organometallic chemistry. Monograph. - American Chemical Society, 1960. - $631 \mathrm{p}$.

20. Barder T.J., Walton R.A., Cotton F.A., Powell G.L. Tetrabutylammonium Octachlorodirhenate(III). // Inorg. Synth. - 1985. - Vol. 23. - P. 116-118.

21. Golichenko A.A., Shtemenko A.V., Kogura O.V. New methods of synthesis the isomer halocarboxylates of dirhenium(III). // Voprosy khimii I khimicheskoi technologii. - 2001. - Vol. 6. - P. 14-16.

22. Cotton F.A., Curtis N., Johnson B., Robinson W. Compounds containing dirhenium(III) octahalide anions. // Inorg. Chem. - 1965. - Vol. 4. - P. 326-330. https://doi.org/10.1021/ic50025a014

23. Shtemenko A.V., Bovykin B.A. Chemistry of binuclear Rhenium Clusters. Rhenium and rhenium allays. - Pennsylvania: TMS publications, 1967. - pp. 189-197.

24. [otton F.A., Freuz B.A., Stults B.R., Webb T.R. Investigations of quadruple bonds by polarized crystal spectra. I. The interpretation of the spectrum of tetra(n-butylammonium) octachlorodirhenate. The disorded crystal structure. // J. Am. Chem. Soc. - 1976. - Vol. 98. - P. 2768-2773. https://doi.org/10.1021/ja00426a016

25. Trogler W.C., Cowman C.D., Gray H.B., Cotton F.A. Further studies of the electronic spectra of $\mathrm{Re}_{2} \mathrm{Cl}_{8}^{2-}$ and $\mathrm{Re}_{2} \mathrm{Br}_{8}{ }^{2-}$. Assignment of the weak bands in the $600-350 \mathrm{~nm}$ region. Estimation of the dissociation energies of Metal-Metal quadruple bonds. // J. Am. Chem. Soc. - 1977. - Vol. 99. - P. 2993-2996. https://doi.org/10.1021/ ja00451a023

26. Cowman C.D., Gray H.B. Low-temperature polarized spectral study of the lowest electronic absorption band in $\mathrm{Re}_{2} \mathrm{Cl}_{8}{ }^{2-}$ and related binuclear complexes. // J. Am. Chem. Soc. - 1973. - Vol. 95. - P. 8177-8188. https://doi. org/10.1021/ja00805a042

Стаття надійшла до редакції 26.01.2018

\section{М. С. Изюмский, А. В. Штеменко}

ГВУЗ «Украинский государственный химико-технологический университет», кафедра неорганической химии,

пр. Гагарина, 8, г. Днепропетровск, 49005, Украина

\section{РАДИКАЛЬНЫЙ МЕХАНИЗМ ОБРАЗОВАНИЯ Сu-Re И Pb-Re КОМПОЗИТОВ ИЗ ГАЗОВОЙ ФАЗЫ ПУТЕМ ТЕРМОРАСПАДА ТРАНС-ТЕТРАХЛОРО-ДИ-}

\section{Резюме}

Исследованы реакции термической деструкции в динамической инертной атмосфере индивидуального транс-тетрахлоро-ди-ц-пропионата дирения(III). Композиты $\mathrm{Cu}-\mathrm{Re}$ и $\mathrm{Pb}-\mathrm{Re}$ на керамике получены газофазной радикальной транспортной реакцией. Сво- 
бодные радикалы $\mathrm{C}_{2} \mathrm{H}_{5}$ - обнаружены по реакции с металлическими зеркалами $\mathrm{Cu}$ и $\mathrm{Pb}$. Композиты $\mathrm{Cu}-\mathrm{Re}$ и $\mathrm{Pb}-\mathrm{Re}$ изучены методами РФА, микрорентгеноспектральным анализом и растровой электронной микроскопией. $\mathrm{Cu}-\mathrm{Re}$ композит состоит из нано дендритов меди, которые «ростут» из рениевой основы, и имеют размеры от 100 нм до 1 мкм, также возможно что они имеют структуру нанотрубок.

Ключевые слова: рений, свободный радикал, карбоксилат, композит, механизм, разложение.

\title{
М. С. Ізюмський, О. В. Штеменко
}

ДВУЗ «Український державний хіміко-технологічний університет», кафедра неорганічної хімії, пр. Гагаріна 8, м. Дніпропетровськ, 49005, Україна

\section{РАДИКАЛЬНИЙ МЕХАНІЗМ УТВОРЕННЯ Cu-Re TA Pb-Re КОМПОЗИТІВ 3 ГАЗОВОЇ ФАЗИ ШЛЯХОМ ТЕРМОРОЗКЛАДУ ТРАНС-ТЕТРАХЛОРО-ДИ-}

\begin{abstract}
Резюме
Досліджено реакції термічної деструкції в динамічній інертній атмосфері індивідуального транс-тетрахлоро-ди- $\mu$-пропіонату диренію(III) та встановлено якісним хімічним аналізом утворення металічного ренію, хлороводню, карбон(IV) оксиду, первинних та вторинних галогеноалкілів. Первинні галогеноалкіли визначено нагріванням 3 натрій тіосульфатом за зміною кольору на синій паперу змоченого сумішшю індикатору конго з гідроген пероксидом. Вторинні галогеноалкіли визначалися взаємодією $35 \mathrm{~N}$ натрій гідроксидом та зміною кольору шару піридином на світло-рожевий. Кількість утвореного металічного ренію складає $99,1 \%$ від вмісту у вихідному транс-тетрахлороди- $\mu$-пропіонаті диренію(III), що визначено кількісним аналізом, шляхом розчинення металічного ренію у концентрованому гідроген пероксиді та подальшому титруванні утвореної перренатної кислоти розчином $0,1 \mathrm{~N}$ натрій гідроксиду з індикатором фенолфталеїном. Композити $\mathrm{Cu}-\mathrm{Re}$ та $\mathrm{Pb}-\mathrm{Re}$ на кераміці одержано у вигляді дзеркал газофазною радикальною транспортною реакцією, через взаємодію транс-тетрахлоро-ди$\mu$-пропіонату диренію(III) з компактними міддю та свинцем. Вільні радикали $\mathrm{C}_{2} \mathrm{H}_{5}$ в виявлено за реакцією з металічними дзеркалами чистих $\mathrm{Cu}$ та $\mathrm{Pb}$. Утворення леткої сполуки $\mathrm{Pb}\left(\mathrm{C}_{2} \mathrm{H}_{5}\right)_{4} \uparrow$ або $\mathrm{Pb}\left(\mathrm{C}_{2} \mathrm{H}_{5}\right)_{\mathrm{n}} \uparrow$ доведено здатністю реакційного газу з установки, при пропусканні через розчин калій йодиду утворювати жовтий осад плюмбум(II) йодиду, який визначено рентгенофазовим аналізом. Взаємодія реакційного газу з калій йодидом доводить утворення летких алкільних сполук плюмбуму при взаємодії з транстетрахлоро-ди- $\mu$-пропіонатом диренію(III), та показує радикальний механізм терморозкладу. Склад та морфологію поверхні композитів $\mathrm{Cu}-\mathrm{Re}$ та $\mathrm{Pb}-\mathrm{Re}$ охарактеризовано методами ренгенофазового та мікрорентгеноспектрального аналізів і растровою електронною мікроскопією. Cu-Re композит складається з нанодендритів міді, які «ростуть» з ренієвої основи, та мають розміри від 100 нм до 1 мкм, також можливо що вони мають структуру нанотрубок.
\end{abstract}

Ключові слова: реній, вільний радикал, карбоксилат, композит, механізм, розклад.

\section{REFERENCES}

1. Kotel'nicova A.S., Tronev V.G. Research the complexes of rhenium(II). Zh. Neorg. Khim., 1958, vol. 3, pp. 1008-1027.

2. Cotton F.A. Metal-Metal Bonding in $\left[R_{2} X_{8}\right]^{2-}$ Ions and Other Metal Atom Clusters. Inorg. Chem., 1965, vol. 4, pp. 334-336. https://doi.org/10.1021/ic50025a016 
3. Cotton F.A., Murillo C.A., Walton R.A. Multiple bonds between metal atoms. New York, Springer, 2005 , 818 p.

4. Steblevsky A.V., Alihanian A.S., Vedenkina L.G. Mass spectral investigation processes of sublimation some rhenium cluster compounds. Russ. J. Coord. Chem., 1984, vol. 10, pp. 72-76.

5. Iziumskyi M., Melnyk S., Shtemenko A. Polymetallic Copper-Rhenium Composite Material. Chem. Met. Alloys., 2013, vol. 6, no 3/4, pp. 121-124.

6. Iziumskyi M., Baskevich A., Melnyk S., Shtemenko A. Thermodynamic properties of trans-tetrachlorodi- $\mu$ carboxylate dirhenium(III) complexes. New. J. Chem., 2016, vol. 40, pp. 10012-10015. https://doi.org/10.1039/ C6NJ02393B

7. Shtemenko A.V., Bovykin B.A., Shram B.C. Thermal decomposition of rhenium binuclear halocarboxylates. Zh. Neorg. Khim., 1985, vol. 12, no 30, pp. 3085-3089.

8. Borisova L.V., Ermakov A.N. Analytical chemistry of rhenium [Analiticheskaja himija renija]. Moscow, Science, 1974, 319 p. (in Russian)

9. Feigl F. Spot tests in organic analysis (6th ed.). New York, Elsevier, 1960, 836 p.

10. [Rozancev E.G., Sholle V.D. Organic chemistry of free radicals [Organicheskaja himija svobodnyh radikalov]. Moscow, Chemistry, 1979, 344 p. (in Russian)

11. Cotton F.A., Wilkinson G. Advanced inorganic chemistry. Willey, 1969, 592 p.

12. Cotton F.A., Wilkinson G. Basic inorganic chemistry. Willey, 1976, 678 p.

13. Green M. Organometallic compounds. The transition elements. Vol. 2, London, 1968, 457 p.

14. Kostromina N.A., Kumok V.N., Skorik N.A. Chemistry of coordination compounds [Himija koordinacionnyh soedinenij]. Moskow, High School, 1990, 432 p. (in Russian)

15. Alekseev S.O. Chemistry of complex compounds [Himija kompleksnyh soedinenij]. Kiev, 2010, 159 p. (in Ukrainian)

16. Lawrance G.A. Introduction to coordination chemistry. Willey, 2010, $290 \mathrm{p}$.

17. Pohodenko V.D., Beloded A.A., Koshechko V.G. Redox reactions of free radicals [Redoks reakcii svobodnyh radikalov]. Kiev, Scientific idea, 1977, 277 p. (in Russian)

18. Sokolov M.N., Samsonenko D.G. Coordination chemistry. Part II: Metalorganic compounds, catalysis with complexes of transition metals, cluster compounds [Koordinacionnaja himija. Chast II: metaloorganicheskie soedinenija, kataliz s kompleksami perehodnyh metallov, klasternye soedinenija]. Novosibirsk, 2011, 194 p. (in Russian)

19. Zeiss H. Organometallic chemistry. Monograph. American Chemical Society, 1960, 631 p.

20. Barder T.J., Walton R.A., Cotton F.A., Powell G.L. Tetrabutylammonium Octachlorodirhenate(III). Inorg. Synth., 1985, vol. 23, pp. 116-118.

21. Golichenko A.A., Shtemenko A.V., Kogura O.V. New methods of synthesis the isomer halocarboxylates of dirhenium(III) [Novye metody sinteza izomernyh halokarboksilatov direnija(III)]. Voprosy khimii i khimicheskoi technologii (Issues of chemistry and Chemical Technology), 2001, vol. 6, pp. 14-16. (in Russian)

22. Cotton F.A., Curtis N., Johnson B., Robinson W. Compounds containing dirhenium(III) octahalide anions. Inorg. Chem., 1965, vol. 4, pp. 326-330. https://doi.org/10.1021/ic50025a014

23. Shtemenko A.V., Bovykin B.A. Chemistry of binuclear Rhenium Clusters. Rhenium and rhenium allays. Pennsylvania, TMS publications, 1967, pp. 189-197.

24. Cotton F.A., Freuz B.A., Stults B.R., Webb T.R. Investigations of quadruple bonds by polarized crystal spectra. I. The interpretation of the spectrum of tetra(n-butylammonium) octachlorodirhenate. The disorded crystal structure. J. Am. Chem. Soc., 1976, vol. 98, pp. 2768-2773. https://doi.org/10.1021/ja00426a016

25. Trogler W.C., Cowman C.D., Gray H.B., Cotton F.A. Further studies of the electronic spectra of $\mathrm{Re}_{2} \mathrm{Cl}_{8}{ }_{8}^{2-}$ and $\operatorname{Re}_{2} \mathrm{Br}_{8}{ }^{2-}$. Assignment of the weak bands in the 600-350 $\mathrm{nm}$ region. Estimation of the dissociation energies of Metal-Metal quadruple bonds. J. Am. Chem. Soc., 1977, vol. 99, pp. 2993-2996. https://doi.org/10.1021/ ja00451a023

26. Cowman C.D., Gray H.B. Low-temperature polarized spectral study of the lowest electronic absorption band in $\mathrm{Re}_{2} \mathrm{Cl}_{8}{ }^{2-}$ and related binuclear complexes. J. Am. Chem. Soc., 1973, vol. 95, pp. 8177-8188. https://doi. org/10.1021/ja00805a042 